Received: 25 January 2019

Accepted: 4 July 2019

Published online: 13 August 2019

\section{Novel and traditional lipid profiles in Metabolic Syndrome reveal a high atherogenicity}

Sílvia Paredes ${ }^{1}$, Liliana Fonseca ${ }^{2}$, Laura Ribeiro ${ }^{3,4}$, Helena Ramos ${ }^{5}$, José Carlos Oliveira ${ }^{6}$ \& Isabel Palma ${ }^{7}$

Low-density-lipoprotein cholesterol (LDL-c) guides lipid-lowering therapy, although other lipid parameters could better reflect cardiovascular disease (CVD) risk. Discordance between these parameters and LDL-c has not been evaluated in metabolic syndrome (MetS) patients. We characterized a comprehensive lipid profile in 177 MetS patients. The 2016 ESC/EAS Guidelines for the Management of Dyslipidemias were used to define LDL-c targets. The atherogenic lipoprotein profile was compared in patients with LDL-c within and above the target. Only $34.4 \%$ (61) of patients had mean LDL-c levels within the guidelines and patients with LDL-c above target presented significantly elevated levels of Apolipoprotein B (ApoB), non-high-density lipoprotein cholesterol (non-HDL-c) and oxidized LDL-c. In patients with LDL-c within target, $25 \%, 31 \%$ and $49 \%$ presented levels above the recommended range for ApoB, non-HDL-C and oxidized LDL-C, respectively. Patients presented a strong association of LDL-C and non-HDL-c $(r=0.796)$, ApoB $(r=0.749)$ and oxidized LDL-c $(r=0.452)$. Similarly, non-HDL-c was strongly correlated with ApoB $(r=0.857)$ and oxidized-LDL-c $(r=0.555)$. The logistic regression model evidenced higher triglycerides and HDL-c and lower ApoB as predictors of having LDL-c within target. Reliance solely on LDL-c could result in missed opportunities for CVD risk reduction. ApoB, oxidized LDL-C, and particularly non-HDL-c, could be valuable parameters to estimate the CVD risk of MetS patients and have the potential to be targeted therapeutically.

The importance of low-density lipoprotein (LDL) cholesterol (LDL-c) reduction to prevent cardiovascular (CV) disease (CVD) is strongly acknowledged ${ }^{1,2}$. Although LDL-c is recommended as the primary marker to guide lipid-lowering therapy ${ }^{3}$, some studies have suggested that other lipid parameters may better represent coronary heart disease (CHD) risk $^{4,5}$. In fact, the 2016 European Society of Cardiology /European Atherosclerosis Society (ESC/EAS) Guidelines for the management of dyslipidemias advocates that non-high-density lipoprotein cholesterol (non-HDL-c) and Apolipoprotein B (ApoB) should be evaluated and considered secondary targets for lipid control $^{3}$. This is in accordance with a growing body of evidence suggesting that other lipid parameters such as non-HDL-c or ApoB may provide a more accurate measure of CHD risk in comparison to LDL-c $\mathrm{c}^{4,5}$.

Patients with metabolic syndrome (MetS), a group at high risk of developing CVD, show several lipid abnormalities other than elevated LDL-c, namely elevated triglycerides levels, low high-density lipoprotein (HDL) cholesterol (HDL-c) and high levels of small and dense LDL (sd-LDL) particles ${ }^{6}$. Thus, evidence suggests that measuring LDL-c may be insufficient in these patients.

The aim of this study was to evaluate a comprehensive lipid profile in MetS patients and compare those achieving and not achieving LDL-c control in respect to other non-conventional lipid parameters.

${ }^{1}$ Sílvia Cristina de Sousa Paredes. Endocrinology Department, Hospital de Braga, Sete Fontes, São Victor, 4710-243, Braga, Portugal. ${ }^{2}$ Liliana Cecília Martins da Fonseca. Endocrinology Department, Centro Hospitalar e Universitário do Porto, Largo Professor Abel Salazar, 4099-001, Porto, Portugal. ${ }^{3}$ Laura Virgínia Pereira Teixeira Ribeiro. Department of Public Health and Forensic Sciences, and Medical Education, Medical Education Unit, Faculty of Medicine of the University of Porto, 4200-319, Porto, Portugal. ${ }^{4}$ I3S-Instituto de Investigação e Inovação em Saúde, Universidade do Porto, 4200-319, Porto, Portugal. ${ }^{5}$ Maria Helena da Silva Ramos. Endocrinology Department, Centro Hospitalar

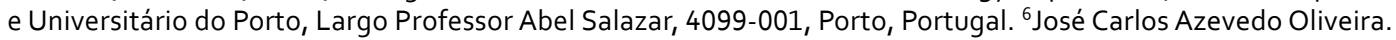
Clinical Chemistry Department, Centro Hospitalar e Universitário do Porto, Largo Professor Abel Salazar, 4099-001, Porto, Portugal. ${ }^{7}$ Isabel Maria Gonçalves Mangas Neto da Palma. Endocrinology Department, Centro Hospitalar e Universitário do Porto, Largo Professor Abel Salazar, 4099-001, Porto, Portugal. Correspondence and requests for materials should be addressed to S.P. (email: silvia.sparedes@gmail.com) 


\section{Materials and Methods}

Subjects and study design. We performed a retrospective data evaluation of our institution's database and electronic medical records system for patients included in the outpatient department at a Portuguese University Hospital, with information regarding an extended lipid profile. Patients followed in the Dyslipidemia and Dermatology outpatient departments between January 2010 and September 2017 were included. After analysis of the medical records of 345 patients, 177 patients with the diagnosis of MetS were included in this study.

Data collection. Obesity was defined as a body mass index (BMI) $\geq 30 \mathrm{~kg} / \mathrm{m}^{2}$ and overweight as BMI $\geq 25$ and $<30 \mathrm{~kg} / \mathrm{m}^{27}$. Hypertension was defined as systolic blood pressure $\geq 140 \mathrm{mmHg}$ and/or diastolic blood pressure $\geq 90 \mathrm{mmHg}$ on at least two blood pressure measurements per visit and on at least two visits ${ }^{8}$ and/or prescription of any antihypertensive medication. Diabetes was defined as plasma glucose $\geq 126 \mathrm{mg} / \mathrm{dL}$ in at least two measurements, A1c $\geq 6.5 \%$ or prescription of any antidiabetic medication ${ }^{9}$. Prediabetes was defined as a plasma glucose $\geq 100$ and $<126 \mathrm{mg} / \mathrm{dL}^{9}$. MetS was classified using the 2009 update: abdominal obesity (waist circumference $\geq 94 \mathrm{~cm}$ for men and $\geq 80 \mathrm{~cm}$ for women $)^{10}$; triglycerides $\geq 150 \mathrm{mg} / \mathrm{dL}$ or the presence of drug treatment for elevated triglycerides; HDL-c $<40 \mathrm{mg} / \mathrm{dL}$ in men and $<50 \mathrm{mg} / \mathrm{dL}$ in women or the presence of a drug treatment for reduced HDL-c; systolic blood pressure $\geq 130 \mathrm{mmHg}$ and/or diastolic blood pressure $\geq 85 \mathrm{mmHg}$ or the presence of an antihypertensive drug; fasting glucose $\geq 100 \mathrm{mg} / \mathrm{dL}$ or the presence of a lowering-glucose drug ${ }^{10}$. Any 3 of 5 risk factors constitutes a diagnosis of $\mathrm{MetS}^{10}$.

Patients were classified as smokers if they consumed any quantity of tobacco. A former smoker was defined as having quit smoking at least 6 months before assessment. Alcohol consumption was considered when patients reported drinking daily at least 1 or 2 glasses of an alcoholic beverage, for women and men respectively.

Patients with conditions that could interfere with the lipid profile, such as genetic dyslipidemia, hepatic or renal moderate to severe disease, cancer, viral infection (hepatitis B or C, HIV), genetic metabolic disease, hyper or hypothyroidism were excluded. Pediatric patients and pregnant women were also excluded as well as patients treated with drugs that could exacerbate lipid profile, such as glucocorticoids.

In order to stratify risk categories, patients were classified as having "very high risk", "high risk", "moderate risk" or "low risk" accordingly to the 2016 ESC/EAS Guidelines for the Management of Dyslipidemias. . The 10-year risk of fatal CVD was calculated using Systemic Coronary Risk Estimation (SCORE). LDL-c targets were defined according to the following ESC/EAS Guidelines ${ }^{3}$, LDL-c $<70 \mathrm{mg} / \mathrm{dL}$ for very high-risk patients, LDL-c $<100 \mathrm{mg} / \mathrm{dL}$ for high risk and LDL-c $<115 \mathrm{mg} / \mathrm{dL}$ for low to moderate risk. In order to analyze other lipid parameters and their agreement with LDL-c levels, targets were also defined for these parameters. The 2016 ESC/EAS Guidelines for the Management of Dyslipidemias was used to define targets such as ApoB, Apolipoprotein A1 (ApoA1), non-HDL-c and Lipoprotein (a) $[\mathrm{Lp}(\mathrm{a})]^{3}: \mathrm{ApoB}:<80 \mathrm{mg} / \mathrm{dL}$ in very high risk individuals and $<100 \mathrm{mg} / \mathrm{dL}$ in high risk individuals; Non-HDL-c: $<100 \mathrm{mg} / \mathrm{dL}$ in very high risk individuals, $<130 \mathrm{mg} / \mathrm{dL}$ in high risk individuals and $<145 \mathrm{mg} / \mathrm{dL}$ in low to moderate risk individuals; ApoA1: low if $<120 \mathrm{mg} / \mathrm{dL}$ in men and $<140 \mathrm{mg} / \mathrm{dL}$ in women; $\mathrm{Lp}(\mathrm{a})<50 \mathrm{mg} / \mathrm{dL}$. For the remaining parameters, the following laboratory reference values were used: oxidized LDL-c: $26-117 \mathrm{mg} / \mathrm{dL}$; ApoB/ApoA1 ratio: 0.45-1.25 and sd-LDL particles: $<35 \mathrm{mg} / \mathrm{dL}$.

Laboratory analysis. Biochemical laboratory tests were conducted after an 8-hour night fast. Lipid parameters included total cholesterol, HDL-c, triglycerides, ApoB, ApoA1, oxidized LDL-c, Lp(a) and sd-LDL. LDL-c levels were calculated through Friedewald's formula ${ }^{11}$ : LDL-c $(\mathrm{mg} / \mathrm{dL})=$ total cholesterol $(\mathrm{mg} / \mathrm{dL})-\mathrm{HDL}-\mathrm{c}$ $(\mathrm{mg} / \mathrm{dL})$ - triglycerides $(\mathrm{mg} / \mathrm{dL}) / 5$, unless triglycerides $\geq 400 \mathrm{mg} / \mathrm{dL}$, where direct LDL-c measurement was performed. Non-HDL-c was calculated by subtracting HDL-c to total cholesterol. Other parameters such as creatinine, uric acid, glucose and homocysteine were also evaluated. Alc was assessed in the majority of diabetic patients. Creatinine, glucose, uric acid, total cholesterol, HDL-c and triglycerides were measured using an enzymatic colorimetric method, with intra and inter-assay coefficients of variation of $<2.8 \%$ and $<3.9 \%$, respectively. These parameters were measured using an automated autoanalyzer (Cobas 8000, Roche Diagnostics, Mannheim, Germany). ApoA1, ApoB and Lp(a) were evaluated through immunoturbidimetry, with intra and inter-assay coefficients of variation of $<1 \%$ and $<2.4 \%$ for ApoA $1,<1.2 \%$ and $<3.2 \%$ for ApoB and $<3.7 \%$ and $<3.8 \%$ for Lp(a), respectively. These parameters were evaluated using an automated autoanalyzer (Cobas Integra 400, Roche Diagnostics, Mannheim, Germany). Oxidized LDL-c was measured by Sandwich Enzyme-Linked ImmunoSorbent Assay (Mercodia, Uppsala, Sweden), with intra and inter-assay coefficients of variation of $<7.3 \%$ and $<8.3 \%$. Sd-LDL particles were measured using an enzymatic colorimetric method (Randox Laboratories, UK) with intra and inter-assay coefficients of variation of $<3 \%$ and $<3 \%$. Alc was evaluated using an affinity chromatography method (Variant II turbo, BioRad Laboratories, CA, USA), with intra and inter-assay coefficients of variation of $<0.78 \%$ and $<0.66 \%$. Homocysteine was measured by nephelometry (Dimension Vista 500 , Siemens Healthcare, Germany), with intra and inter-assay coefficients of variation of $<3.3 \%$ and $<8.2 \%$.

Statistical analysis. Statistical analysis was performed using IBM SPSS ${ }^{\circledR}$ version 25.0 and a p value below 0.05 was considered statistically significant. For continuous quantitative variables, distribution normality was tested through histogram observation and kurtosis and skewness analysis. Results are presented as mean values \pm standard-deviation and median values (25-75 percentiles). The chi-square test was used to analyze differences between groups in categorical variables. The Student t-test for independent variables and the Mann Whitney test were used to compare continuous variables with normal and non-normal distribution between groups, respectively. Correlations were evaluated using the Pearson and the Spearman correlation tests for continuous symmetrical and asymmetrical variables, respectively, and applying a Bonferroni correction. A logistic regression model was performed in order to evaluate predictors of LDL-c within target, adjusting for potential confounders using a stepwise regression with a forward inclusion approach. 


\begin{tabular}{|c|c|c|c|c|c|}
\hline & $\mathbf{n}$ & $\begin{array}{l}\text { LDL-c within } \\
\operatorname{target}(n=61)\end{array}$ & $\mathbf{n}$ & $\begin{array}{l}\text { LDL-c above target } \\
(\mathrm{n}=116)\end{array}$ & $p$ \\
\hline Female n (\%) & 61 & $25(41 \%)$ & 116 & $53(45.7 \%)$ & 0.549 \\
\hline Age (years) & 61 & $54.6 \pm 13.1$ & 116 & $54.1 \pm 11.7$ & 0.790 \\
\hline Body mass index $\left(\mathrm{Kg} / \mathrm{m}^{2}\right)$ & 47 & $29.9 \pm 3.8$ & 93 & $30.5 \pm 4.8$ & 0.465 \\
\hline Systolic BP (mmHg) & 56 & $140.0 \pm 17.0$ & 102 & $138.2 \pm 18.5$ & 0.535 \\
\hline Diastolic BP (mmHg) & 45 & $79.4 \pm 12.6$ & 74 & $80.8 \pm 13.6$ & 0.571 \\
\hline Waist circumference $(\mathrm{M})(\mathrm{cm})$ & 19 & $103.5 \pm 9.0$ & 23 & $105.0 \pm 11.2$ & 0.653 \\
\hline Waist circumference $(\mathrm{F})(\mathrm{cm})$ & 10 & $104.4 \pm 13.6$ & 23 & $100.3 \pm 9.0$ & 0.310 \\
\hline Triglycerides* $(\mathrm{mg} / \mathrm{dL})$ & 61 & $174.0(122.5-302.5)$ & 116 & $165.5(110.0-239.2)$ & 0.299 \\
\hline HDL-c (M) (mg/dL) & 36 & $35.8 \pm 10.3$ & 63 & $39.2 \pm 10.3$ & 0.117 \\
\hline HDL-c (F) (mg/dL) & 25 & $44.9 \pm 11.4$ & 53 & $43.2 \pm 12.1$ & 0.545 \\
\hline Glucose* (mg/dL) & 55 & $102.0(90.0-125.0)$ & 103 & $111.0(94.0-156.0)$ & 0.062 \\
\hline Alc (\%) & 33 & $6.5 \pm 1.6$ & 65 & $7.4 \pm 1.8$ & 0.024 \\
\hline Uric acid $(\mathrm{mg} / \mathrm{dL})$ & 41 & $5.5 \pm 1.7$ & 73 & $5.4 \pm 1.7$ & 0.820 \\
\hline Homocysteine $(\mu \mathrm{mol} / \mathrm{L})$ & 47 & $15.8 \pm 10.3$ & 92 & $13.5 \pm 6.5$ & 0.171 \\
\hline Diabetes n (\%) & 60 & $27(45 \%)$ & 115 & $70(60.9 \%)$ & 0.045 \\
\hline Statin use n (\%) & 56 & $37(66.1 \%)$ & 100 & $57(57 \%)$ & 0.267 \\
\hline Fibrates use $\mathrm{n}(\%)$ & 56 & $14(25 \%)$ & 100 & $14(14 \%)$ & 0.086 \\
\hline Ezetimibe use n (\%) & 61 & $4(6.5 \%)$ & 115 & $7(6.1 \%)$ & 0.596 \\
\hline Alcohol drinking $\mathrm{n}(\%)$ & 36 & $11(30.5 \%)$ & 63 & $18(28.6 \%)$ & 0.817 \\
\hline Smoking n (\%) & 54 & $10(18.5 \%)$ & 102 & $16(15.7 \%)$ & 0.897 \\
\hline
\end{tabular}

Table 1. Comparison of clinical and laboratory variables between MetS patients with LDL-c within and above target. Data are presented as mean \pm standard deviation, unless otherwise indicated by $*$ corresponding to data presented as median, $25^{\text {th }}$ and $75^{\text {th }}$ percentiles. LDL-c $=$ Low-density lipoprotein cholesterol; $\mathrm{BP}=\mathrm{Blood}$ pressure; HDL-c = High-density lipoprotein cholesterol, $\mathrm{M}=$ male; $\mathrm{F}=$ female

Ethics. This study was approved by the local Ethics committee (150-DEFI/149-CES) and procedures were carried out in accordance with the principles contained in the Declaration of Helsinki. Due to the retrospective nature of this study, consent to participate was waived by the Ethics Committee.

\section{Results}

This study enrolled 177 subjects (99 men and 78 women) with a mean age of $54 \pm 12$ years. The clinical characteristics of the patients are presented in Table 1. Mean LDL-c levels were $106.4 \pm 43.4 \mathrm{mg} / \mathrm{dL}$. Diabetes was present in 97 individuals $(54.8 \%)$ and the majority of patients $(n=94,53.1 \%)$ were under lipid-lowering drugs and statins were the most common. Twenty-eight (15.8\%) individuals were on fibrates and $11(6.2 \%)$ on ezetimibe. Patients were not taking other drugs for treating dyslipidemia nor any anti-diabetic drugs that could affect the lipid profile such as thiazolidinediones, glucagon-like peptide- 1 agonists, dipeptidyl peptidase 4 inhibitors or sodium-glucose co-transporter-2 inhibitors. According to the ESC/EAS guidelines, patients were divided in two groups: patients with LDL-c levels within target $(n=61)$ and patients with LDL-c levels above target $(n=116)$. There were no statistically significant differences between the groups regarding gender, age and BMI or between the different features of MetS, namely blood pressure, waist circumference and triglycerides, HDL-c or glucose levels. We found no statistically significant differences between groups in relation to alcohol ingestion, smoking or the use of drugs for dyslipidemia such as statins, fibrates or ezetimibe. Uric acid levels, usually elevated in patients with MetS, and homocysteine levels, a significant predictor of new CV events, were not statistically different between the groups. There was a significant difference between groups with respect to the presence of diabetes.

The lipid profile of MetS patients with LDL-c within and above target is presented in Table 2. Mean LDL-c concentration was $71.4 \pm 22.6 \mathrm{mg} / \mathrm{dL}$ in patients with LDL-c within target and $124.9 \pm 40.3 \mathrm{mg} / \mathrm{dL}$ in those with LDL-c above target $(p<0.001)$. The latter group also presented statistically significant elevated levels of total cholesterol, ApoB, non-HDL-c, ApoB/ApoA1 ratio and oxidized LDL-c. No statistically significant differences were found between the two groups regarding ApoA1 and sd-LDL particles.

Despite having a LDL-c within the goal defined by the guidelines, these patients presented elevated levels of other atherogenic lipoproteins. In fact, $25 \%, 31.1 \%$ and $49.2 \%$ of patients presented ApoB, non-HDL-c and oxidized LDL-c levels, respectively above the threshold for CV risk. LDL-c exhibited positive correlations with several lipid particles. After Bonferroni correction, we found statistically significant positive correlations between LDL- $c$ and total cholesterol $(r=0.813, p<0.001)$, non-HDL-c $(r=0.796, p<0.001)$, ApoB $(r=0.749, p<0.001)$, sd-LDL $(\mathrm{r}=0.629, \mathrm{p}<0.001)$, ApoB/ApoA1 ratio $(\mathrm{r}=0.462, \mathrm{p}<0.001)$ and oxidized-LDL-c $(\mathrm{r}=0.452$, $\mathrm{p}<0.001)$. Non-HDL-c was also significantly and positively correlated with ApoB $(\mathrm{r}=0.857, \mathrm{p}<0.001)$ and oxidized-LDL-c $(r=0.555, \mathrm{p}<0.001)$. The magnitude of this correlation was stronger for non-HDL-c than for LDL-c.

A logistic regression was performed for the identification of variables that could influence LDL-c levels within, or above, target (Table 3). The higher the tryglicerides and the HDL-c levels, the greater the odds of having LDL-c within target, whereas the odds decreased as the ApoB levels increased. 


\begin{tabular}{|l|l|l|l|l|l|}
\hline & $\mathbf{n}$ & LDL-c within target & $\mathbf{n}$ & LDL-c above target & $\boldsymbol{P}$ \\
\hline LDL-c (mg/dL) & 61 & $71.4 \pm 22.6$ & 116 & $124.9 \pm 40.3$ & $<0.001^{\Sigma}$ \\
\hline Total cholesterol (mg/dL) & 61 & $160.6 \pm 41.3$ & 116 & $206.0 \pm 59.9$ & $<0.001^{\Sigma}$ \\
\hline ApoB (mg/dL) & 56 & $80.1 \pm 19.9$ & 107 & $102.9 \pm 33.6$ & $<0.001^{\Sigma}$ \\
\hline ApoA1 (F) (mg/dL) & 21 & $145.5 \pm 34.3$ & 50 & $144.9 \pm 31.5$ & 0.939 \\
\hline ApoA1 (M) (mg/dL) & 32 & $133.8 \pm 35.0$ & 52 & $128.3 \pm 26.0$ & 0.413 \\
\hline ApoB/ApoA1 ratio & 51 & $0.6 \pm 0.2$ & 101 & $0.8 \pm 0.3$ & $<0.001^{\Sigma}$ \\
\hline Non-HDL-c* (mg/dL) & 61 & $115.0(95.5-135.0)$ & 116 & $153.5(123.0-195.7)$ & $<0.001^{\Sigma}$ \\
\hline Oxidized LDL-c*(U/L) & 61 & $115.7(84.8-162.4)$ & 116 & $153.0(112.15-216.9)$ & $<0.001^{\Sigma}$ \\
\hline sd-LDL (mg/dL) & 11 & $28.2 \pm 13.2$ & 14 & $40.7 \pm 19.4$ & 0.079 \\
\hline Lipoprotein (a)*(mg/dL) & 54 & $10.0(4.0-34.5)$ & 103 & $24.0(7.0-55.0)$ & 0.027 \\
\hline
\end{tabular}

Table 2. Comparison of lipid profile between MetS patients with LDL-c within and above target. Data are presented as mean \pm standard deviation, unless otherwise indicated by $*$ corresponding to data presented as median, $25^{\text {th }}$ and $75^{\text {th }}$ percentiles. The symbol $\sum$ represent data that maintain statistical significance after Bonferroni correction. LDL-c = Low-density lipoprotein cholesterol; ApoB = Apolipoprotein B; ApoA1 = Apolipoprotein A1; $\mathrm{M}=$ male; $\mathrm{F}=$ female; Non-HDL-c = non-nigh-density lipoprotein cholesterol; sd-LDL $=$ small and dense LDL-c

\begin{tabular}{|l|l|l|l|}
\hline & OR & $\mathbf{9 5 \%}$ CI & $\boldsymbol{p}$ \\
\hline Triglycerides & 1.011 & $1.003-1.020$ & 0.010 \\
\hline HDL-c & 1.178 & $1.011-1.373$ & 0.035 \\
\hline ApoB & 0.883 & $0.813-0.959$ & 0.003 \\
\hline
\end{tabular}

Table 3. Predictors of LDL-c within target. Included covariables: age, gender, waist circumference, systolic blood pressure, dyastolic blood pressure, presence of diabetes, presence of obesity, statin use, fibrate use, ApoA1, glucose; HDL-c = High density lipoprotein cholesterol; ApoB = Apolipoprotein B; OR = Odds Ratio; 95\% $\mathrm{CI}=95 \%$ Confidence interval

\section{Discussion}

As CVD is the most common cause of death in the developed world ${ }^{12}$, early identification of individuals at increased CV risk is a priority. Global guidelines recommend LDL-c as the cornerstone of lipid management despite other lipid parameters gaining momentum as indexes for CV risk. Also, the clinical expression of the MetS dyslipidemia has a different pattern since hypercholesterolemia is not the main feature. The present study shows that in MetS several atherosclerotic lipoproteins remain elevated even when the patients have optimal LDL-c levels. Thus, in these patients, the LDL-c measurement may be insufficient to estimate CV risk.

Non-HDL-C and ApoB. We found that $31 \%$ and $25 \%$ of MetS individuals had high non-HDL-c and ApoB levels, respectively, regardless of having an LDL-c below the cut off for which treatment initiation is recommended. Since both non-HDL-c and ApoB are considered "secondary targets", these parameters may not be routinely evaluated, after LDL-c control. Several studies have demonstrated a stronger association for non-HDL-c and ApoB vs LDL-c with CHD events ${ }^{13,14}$, also in MetS patients ${ }^{15}$. Moreover, ApoB and non-HDL-c have been closely associated with MetS development ${ }^{15,16}$ and their levels are higher in MetS patients compared to those without MetS, regardless of the LDL-c level ${ }^{5,17}$. In line with this, the majority of our patients presented elevated ApoB and non-HDL-c levels. It has been demonstrated that in MetS patients or in those with evidence of insulin resistance, a discordance between ApoB and LDL-c is more evident and ApoB appears to be superior to LDL-c in predicting CV risk ${ }^{18}$. Non-HDL-c has also been suggested as a better marker of CVD risk and coronary atherosclerosis $^{19}$ including MetS patients ${ }^{4}$. Moreover, Kilgore et al. (2014) found that these patients are more likely to have high non-HDL-c with normal LDL-c rather than high LDL-c with normal non-HDL-c ${ }^{4}$. Our results reveal that in MetS patients when only LDL-c is considered, those at a high CV risk may not be identified as candidates for lifestyle changes or pharmacologic lipid-lowering interventions.

ApoA1 and ApoB/ApoA1 ratio. ApoA1 has been suggested to mediate the association between MetS and atherosclerosis ${ }^{20}$. Nonetheless, according to the ESC/EAS guidelines, MetS patients show normal ApoA1 concentrations. In our study, we did not find any differences in ApoA1 levels between patients with LDL-c within and above target. Moreover, our results are not in agreement with others which have found reduced ApoA1 levels in MetS patients ${ }^{21}$. Nevertheless, it seems that not only the concentration but also ApoA1 function may be affected in this condition. In fact, Borja et al. (2017) have found that in MetS patients, HDL-c/ApoA1 exchange was reduced regardless of ApoA1 levels ${ }^{21}$. ApoB/ApoA1 ratio is a simple and accurate risk factor for CVD ${ }^{22,23}$. In our study, despite the fact that the MetS patients presented a mean ApoB/ApoA1 ratio within the reference range, patients with LDL-c levels above target showed a significantly higher ratio than those within target. Carnevale et al. (2011) found that independently from LDL-c, a low ApoB/apoA1 ratio reflects a less atherogenic lipid profile $^{23}$. Also, Wallenfeldt et al. (2004) reported that this ratio was associated with an increase in carotid artery 
intima-media thickness, independently of other risk factors ${ }^{24}$ in MetS patients. Thus, the clinical risk information provided by ApoB/ApoA1 ratio should be recognized.

Oxidized LDL-c. In our study, $49 \%$ of patients with MetS and LDL-c within target had oxidized LDL-c above the reference range. Numerous studies have demonstrated an atherogenic role of oxidized LDL-c in the progression of atherosclerosis ${ }^{25}$ and $\mathrm{CHD}^{26}$. Furthermore, the predictive value of oxidized LDL-c seems to be additive to that of the Framingham global risk assessment score for CV risk prediction ${ }^{26}$. Oxidative stress seems to be involved in the pathophysiology of MetS ${ }^{27}$ and oxidized LDL-c is elevated in MetS patients ${ }^{28}$. In fact, MetS dyslipidemia is characterized by the elevation of the easily oxidized sd-LDL particles ${ }^{29}$. This is in accordance with our results where the MetS patients had generally elevated oxidized LDL-c levels and the patients with LDL-c above target showed even higher levels than those with LDL-c within target. More disturbing is the fact that almost half of the LDL-c within target patients presented oxidized LDL-c levels above the reference range, thus suggesting a high risk of progression of atherosclerotic CVD.

Lp(a) and small and dense LDL particles. Lp(a) is an independent risk factor for $\mathrm{CVD}^{3}$ as well as in MetS individuals ${ }^{30}$. Hippe et al. (2018) found that in MetS patients treated to obtain LDL-c below $70 \mathrm{mg} / \mathrm{dL}$, carotid atherosclerosis progressed and elevated $\mathrm{Lp}$ (a) levels independently predicted this progression ${ }^{31}$. This finding suggests that $\mathrm{Lp}$ (a) can also be a useful marker of atherosclerosis evolution in MetS patients. The association of highly atherogenic Sd-LDL particles ${ }^{32}$ with insulin resistance and hypertriglyceridemia suggests its high prevalence in states such as $\mathrm{MetS}^{33}$. Our results are in agreement with others reporting an increase of sd-LDL particles in MetS patients $^{34,35}$. Although no differences were observed in sd-LDL particles levels between the two groups, patients with LDL-c above target had a mean plasma sd-LDL particles level above $35 \mathrm{mg} / \mathrm{dL}$ indicating a high risk of CHD.

Lipid parameters correlations. As stated before, concomitant dyslipidemia has a particular pattern characterized by a high flux of free fatty acids, hypertriglyceridemia, low HDL-c values, increased sd-LDL particles and high $A$ poB values ${ }^{36,37}$. To the best of our knowledge, no studies have evaluated LDL-c correlations with other lipid parameters according to LDL-c status in patients with MetS. As expected, we observed a positive correlation between LDL-c and total cholesterol and non-HDL-c. In accordance with other authors, we also found a positive association of LDL-c with oxidized LDL-c $\mathrm{c}^{38}$ and with sd-LDL particles ${ }^{39,40}$. Triglycerides affect LDL-c density and thus its propensity to be oxidized whereas HDL-c has a protective role against LDL-c oxidation. Therefore, the atherogenic lipid profile that MetS exhibit can further aggravate oxidative stress in these patients. It is important to note that the presence of sd-LDL particles is affected by other metabolic changes observed in Mets, such as high triglycerides, low HDL-c and insulin-resistance. Non-HDL-c and ApoB were the most strongly correlated with LDL-c. Additionally, non-HDL considered a better risk marker for coronary heart disease exhibited a very strong correlation with ApoB and a strong correlation with oxidized LDL-c. As ApoB and oxidized LDL-c may not be routinely available, the assessment of non-HDL-c could be important to characterize the atherogenic profile. In fact, non-HDL-c is easily obtainable and inexpensive ${ }^{41}$, and represents an appropriate index of CV risk that may be superior to LDL- $\mathrm{c}^{11,42}$. In addition, non-HDL-c is not affected by the association between very-low-density lipoprotein (VLDL) cholesterol and triglycerides which is altered in MetS and leads to falsely low LDL values. On the other hand, non-HDL cholesterol represents the cholesterol carried by all of the potentially atherogenic ApoB-containing particles, i.e., VLDL, intermediate-density lipoprotein (IDL), and LDL, chylomicron remnants and lipoprotein $(a)^{41,42}$. Triglyceride-rich lipoproteins, such as VLDL, IDL and their remnants are highly atherogenic $^{43}$. They can be taken up by arterial wall macrophages directly without oxidative modification and, due to their larger size, they carry more cholesterol per particle than $\mathrm{LDL}^{43}$. Since MetS is characterized by a postprandial accumulation of triglyceride rich lipoproteins ${ }^{43}$, non-HDL-c may be particularly useful for evaluating CVD risk in patients with this condition.

The logistic regression model showed that higher triglycerides and HDL-c levels appear to increase the odds of LDL-c being within target. Leroux et al. (2000) have demonstrated that patients with higher levels of triglycerides are characterized by lower LDL-c levels for any given ApoB concentration compared with subjects with lower levels of triglycerides ${ }^{44}$. An overproduction of the triglycerides-enriched large VLDL causes a high generation of sd-LDL particles that in turn may lead to an overestimation of the VLDL cholesterol and an underestimation of the calculated LDL-c concentrations ${ }^{45}$. Thus, patients with hypertriglyceridemia, such as MetS patients, seem to present lower LDL-c concentrations since these values are falsely diminished by hypertriglyceridemia. Nevertheless, it is important to note that these patients still remain in an environment of high atherogenicity since ApoB remains elevated even when there is a decrease of LDL-c levels ${ }^{44}$. Also, we demonstrated that even when LDL-c levels are optimized, MetS patients still show high levels of important markers of atherogenicity such as non-HDL-c, ApoB and oxidized LDL-c. Cholesterol ester transfer protein is responsible for exchanging the cholesterol esters in the HDL for triglycerides from LDL. Cholesterol ester-rich LDL can return to the liver and be taken up through the LDL receptor ${ }^{46}$. This mechanism may justify the reason why higher HDL-c levels are associated with increased odds of having LDL-c within target. ApoB envelops the surface of several atherogenic lipoproteins ${ }^{47}$ and so greater ApoB values are associated with higher LDL-c levels. MetS patients often present LDL-c/ApoB discordance. In fact, our study suggests that even in patients with "optimal" LDL-c levels ApoB can be elevated.

Limitations. Our study has some limitations. First, this was a cross-sectional study that did not assess long-term outcomes with respect to the occurrence of CVD and the levels of lipid parameters. Thus, prospective follow-up studies are required to evaluate medical interventions and lipid goal attainment in relation to mortality in patients with MetS. Second, few patients had measurements of sd-LDL particles because it was only recently 
available in our hospital. Finally, multiple testing can be associated with type 1 errors, even though a Bonferroni correction was applied to the comparisons to counteract this.

Summary. Our study also has strengths. A small number of studies have assessed non-conventional lipid parameters such as ApoA1, sd LDL particles, Lp(a) or oxidized LDL-c in MetS patients. Our study is an important contribution to the characterization of a comprehensive lipid profile in patients with this condition. Moreover, it also suggests that in MetS patients solely assessing LDL-c could result in missed opportunities for atherosclerotic CVD risk reduction.

\section{Conclusions}

LDL-c reduction has long been the major goal of lipid-modulating therapies; however, patients continue to exhibit CVD-related events. This underscores the need for identification of other predictive lipoprotein parameters. The present study contributes to our understanding of lipid metabolism in MetS patients and it may have important clinical implications. By using LDL-c as the only target, we may be missing significant opportunities to decrease the risk of atherosclerotic CVD. Although further research is needed in this new era, our data suggest that combining LDL-c with non-HDL-c, ApoB and oxidized LDL-c may add important information to better identify therapeutic targets and to stratify CV risk in these patients.

\section{Data Availability}

The datasets generated during and/or analysed during the current study are available from the corresponding author on reasonable request.

\section{References}

1. LaRosa, J. C. et al. Intensive lipid lowering with atorvastatin in patients with stable coronary disease. The New England journal of medicine 352, 1425-1435 (2005).

2. Taylor, F. et al. Statins for the primary prevention of cardiovascular disease. The Cochrane database of systematic reviews, CD004816 (2013).

3. Catapano, A. L. et al. 2016 ESC/EAS Guidelines for the Management of Dyslipidaemias. Eur Heart J 37, 2999-3058 (2016).

4. Kilgore, M. et al. Discordance between high non-HDL cholesterol and high LDL-cholesterol among US adults. J Clin Lipidol 8, $86-93$ (2014)

5. Lim, Y. et al. Apolipoprotein B Is Related to Metabolic Syndrome Independently of Low Density Lipoprotein Cholesterol in Patients with Type 2 Diabetes. Endocrinol Metab (Seoul) 30, 208-215 (2015).

6. Ruotolo, G. \& Howard, B. V. Dyslipidemia of the metabolic syndrome. Curr Cardiol Rep 4, 494-500 (2002)

7. Yumuk, V. et al. European Guidelines for Obesity Management in Adults. Obes Facts 8, 402-424 (2015).

8. Mancia, G. et al. ESH/ESC guidelines for the management of arterial hypertension: the Task Force for the Management of Arterial Hypertension of the European Society of Hypertension (ESH) and of the European Society of Cardiology (ESC). Eur Heart J 34, (2013).

9. American Diabetes, A. 2. Classification and Diagnosis of Diabetes: Standards of Medical Care in Diabetes-2018. Diabetes Care 41, S13-S27 (2018).

10. Alberti, K. G. et al. Harmonizing the metabolic syndrome: a joint interim statement of the International Diabetes Federation Task Force on Epidemiology and Prevention; National Heart, Lung, and Blood Institute; American Heart Association; World Heart Federation; International Atherosclerosis Society; and International Association for the Study of Obesity. Circulation 120, (1640-1645 (2009).

11. Friedewald, W. T., Levy, R. I. \& Fredrickson, D. S. Estimation of the concentration of low-density lipoprotein cholesterol in plasma, without use of the preparative ultracentrifuge. Clin Chem 18, 499-502 (1972).

12. Pagidipati, N. J. \& Gaziano, T. A. Estimating deaths from cardiovascular disease: a review of global methodologies of mortality measurement. Circulation 127, 749-756 (2013).

13. Boekholdt, S. M. et al. Association of LDL cholesterol, non-HDL cholesterol, and apolipoprotein B levels with risk of cardiovascular events among patients treated with statins: a meta-analysis. JAMA 307, 1302-1309 (2012).

14. Sniderman, A. D. et al. A meta-analysis of low-density lipoprotein cholesterol, non-high-density lipoprotein cholesterol, and apolipoprotein B as markers of cardiovascular risk. Circ Cardiovasc Qual Outcomes 4, 337-345 (2011).

15. Boumaiza, I. et al. Apolipoprotein B and non-high-density lipoprotein cholesterol are better risk markers for coronary artery disease than low-density lipoprotein cholesterol in hypertriglyceridemic metabolic syndrome patients. Metab Syndr Relat Disord 8, 515-522 (2010).

16. Ghodsi, S. et al. Non-high-density lipoprotein fractions are strongly associated with the presence of metabolic syndrome independent of obesity and diabetes: a population-based study among Iranian adults. J Diabetes Metab Disord 16, 25 (2017).

17. Huang, J. et al. Non-high-density lipoprotein cholesterol in patients with metabolic syndrome. J Investig Med 56, 931-936 (2008).

18. Varvel, S. A. et al. Discordance between apolipoprotein B and low-density lipoprotein particle number is associated with insulin resistance in clinical practice. J Clin Lipidol 9, 247-255 (2015).

19. Zhang, Y. et al. Non-HDL-C is a Better Predictor for the Severity of Coronary Atherosclerosis Compared with LDL-C. Heart Lung Circ 25, 975-981 (2016).

20. Mattsson, N. et al. Metabolic syndrome and carotid intima-media thickness in young adults: roles of apolipoprotein B, apolipoprotein A-I, C-reactive protein, and secretory phospholipase A2: the cardiovascular risk in young Finns study. Arterioscler Thromb Vasc Biol 30, 1861-1866 (2010).

21. Borja, M. S. et al. Apolipoprotein A-I exchange is impaired in metabolic syndrome patients asymptomatic for diabetes and cardiovascular disease. PLoS ONE 12, e0182217 (2017).

22. Walldius, G. \& Jungner, I. The apoB/apoA-I ratio: a strong, new risk factor for cardiovascular disease and a target for lipid-lowering therapy-a review of the evidence. J Intern Med 259, 493-519 (2006).

23. Carnevale Schianca, G. P. et al. ApoB/apoA-I ratio is better than LDL-C in detecting cardiovascular risk. Nutr Metab Cardiovasc Dis 21, 406-411 (2011).

24. Wallenfeldt, K., Bokemark, L., Wikstrand, J., Hulthe, J. \& Fagerberg, B. Apolipoprotein B/apolipoprotein A-I in relation to the metabolic syndrome and change in carotid artery intima-media thickness during 3 years in middle-aged men. Stroke 35, 2248-2252 (2004).

25. Gao, S. \& Liu, J. Association between circulating oxidized low-density lipoprotein and atherosclerotic cardiovascular disease. Chronic Dis Transl Med 3, 89-94 (2017).

26. Holvoet, P. et al. Association of high coronary heart disease risk status with circulating oxidized LDL in the well-functioning elderly: findings from the Health, Aging, and Body Composition study. Arterioscler Thromb Vasc Biol 23, 1444-1448 (2003). 
27. Rani, V., Deep, G., Singh, R. K., Palle, K. \& Yadav, U. C. Oxidative stress and metabolic disorders: Pathogenesis and therapeutic strategies. Life Sci 148, 183-193 (2016).

28. Holvoet, P. et al. The metabolic syndrome, circulating oxidized LDL, and risk of myocardial infarction in well-functioning elderly people in the health, aging, and body composition cohort. Diabetes 53, 1068-1073 (2004).

29. Hurtado-Roca, Y. et al. Oxidized LDL Is Associated With Metabolic Syndrome Traits Independently of Central Obesity and Insulin Resistance. Diabetes 66, 474-482 (2017).

30. Albers, J. J. et al. Relationship of apolipoproteins A-1 and B, and lipoprotein(a) to cardiovascular outcomes: the AIM-HIGH trial (Atherothrombosis Intervention in Metabolic Syndrome with Low HDL/High Triglyceride and Impact on Global Health Outcomes). J Am Coll Cardiol 62, 1575-1579 (2013).

31. Hippe, D. S. et al. Lp(a) (Lipoprotein(a)) Levels Predict Progression of Carotid Atherosclerosis in Subjects With Atherosclerotic Cardiovascular Disease on Intensive Lipid Therapy: An Analysis of the AIM-HIGH (Atherothrombosis Intervention in Metabolic Syndrome With Low HDL/High Triglycerides: Impact on Global Health Outcomes) Carotid Magnetic Resonance Imaging Substudy-Brief Report. Arterioscler Thromb Vasc Biol 38, 673-678, (2018).

32. Griffin, B. A. Lipoprotein atherogenicity: an overview of current mechanisms. Proc Nutr Soc 58, 163-169 (1999).

33. Watson, K. E., Horowitz, B. N. \& Matson, G. Lipid abnormalities in insulin resistant states. Rev Cardiovasc Med 4, 228-236 (2003).

34. Rizzo, M. \& Berneis, K. Small, dense low-density-lipoproteins and the metabolic syndrome. Diabetes Metab Res Rev 23, 14-20 (2007).

35. Vekic, J. et al. Small, dense LDL cholesterol and apolipoprotein B: relationship with serum lipids and LDL size. Atherosclerosis 207, 496-501 (2009).

36. Kolovou, G. D., Anagnostopoulou, K. K. \& Cokkinos, D. V. Pathophysiology of dyslipidaemia in the metabolic syndrome. Postgrad Med J 81, 358-366 (2005)

37. Bloomgarden, Z. T. Dyslipidemia and the metabolic syndrome. Diabetes Care 27, 3009-3016 (2004).

38. Brizzi, P. et al. Plasma lipid composition and LDL oxidation. Clin Chem Lab Med 41, 56-60 (2003).

39. Hayashi, T. H. T., Shiobara, SuguroT. \& Adachi, M. T. Small dense LDL concentration is closely associated with serum apolipoprotein B, comparisons of non-LDL cholesterol or LDL cholesterol. Rinsho Byori 54, 569-575 (2006).

40. T, H. Metabolic syndrome and small dense LDL-cholesterol. Rinsho Byori 55, 434-438 (2007).

41. Hsia, S. H. Non-HDL cholesterol: into the spotlight. Diabetes Care 26, 240-242 (2003).

42. Lu, W. et al. Non-HDL cholesterol as a predictor of cardiovascular disease in type 2 diabetes: the strong heart study. Diabetes Care 26, 16-23 (2003)

43. Toth, P. P. Triglyceride-rich lipoproteins as a causal factor for cardiovascular disease. Vasc Health Risk Manag 12, 171-183 (2016).

44. Leroux, G. et al. Influence of triglyceride concentration on the relationship between lipoprotein cholesterol and apolipoprotein B and A-I levels. Metabolism 49, 53-61 (2000).

45. Srisawasdi, P. et al. Estimation of plasma small dense LDL cholesterol from classic lipid measures. Am J Clin Pathol 136, 20-29 (2011).

46. Barter, P. J. et al. Cholesteryl ester transfer protein: a novel target for raising HDL and inhibiting atherosclerosis. Arterioscler Thromb Vasc Biol 23, 160-167 (2003).

47. Wilkins, J. T., Li, R. C., Sniderman, A., Chan, C. \& Lloyd-Jones, D. M. Discordance Between Apolipoprotein B and LDL-Cholesterol in Young Adults Predicts Coronary Artery Calcification: The CARDIA Study. J Am Coll Cardiol 67, 193-201 (2016).

\section{Author Contributions}

S.P., L.F., H.R., J.C.O. and I.P. designed the study; S.P. and L.F. acquired the data; S.P., L.F. and L.R. did the data analysis; S.P., L.F., L.R., H.R., J.C.O. and I.P. interpreted the data; S.P., L.F. and L.R. drafted the work and all authors revised it critically for important intellectual content. All authors approved the final version submitted and are accountable for all aspects of the work. All authors read and approved the final manuscript.

\section{Additional Information}

Competing Interests: The authors declare no competing interests.

Publisher's note: Springer Nature remains neutral with regard to jurisdictional claims in published maps and institutional affiliations.

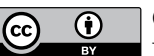

Open Access This article is licensed under a Creative Commons Attribution 4.0 International

License, which permits use, sharing, adaptation, distribution and reproduction in any medium or format, as long as you give appropriate credit to the original author(s) and the source, provide a link to the Creative Commons license, and indicate if changes were made. The images or other third party material in this article are included in the article's Creative Commons license, unless indicated otherwise in a credit line to the material. If material is not included in the article's Creative Commons license and your intended use is not permitted by statutory regulation or exceeds the permitted use, you will need to obtain permission directly from the copyright holder. To view a copy of this license, visit http://creativecommons.org/licenses/by/4.0/.

(c) The Author(s) 2019 